\title{
Les plasmas créés par laser dans le domaine X-UV: application à la "calibration"
}

\author{
R. Benattar
}

PMI et LULI Ecole Polytechnique, 91128 Palaiseau, France

Des expériences d'imagerie et de spectroscopie résolues temporellement ont été réalisées dans le cadre des études sur l'interaction laser-matière, sur des plasmas créés par un laser de puissance focalisé sur cible solide.

Deux types d'expériences ont été développées:

l'étude des effets de préchauffage du milieu froid par le rayonnement issu du plasma

-l'étude du transport par conduction thermique de l'énergie déposée par le laser vers les parties denses du plasma.

Ces études ont montré la nécessité d'étalonner les instruments utilisés.

Nous avons ainsi mis en place un système d'étalonnage d'instruments dans le domaine $\mathrm{X}-\mathrm{UV}$, dans l'intervalle de longueurs d'ondes $40-400 \AA$

Un monochromateur sélectionne le rayonnement en provenance d'un plasma créé par laser. Ce rayonnement monochromatique est envoyé dans une enceinte à vide contenant un goniomètre $\theta / 2 \theta$. A l'aide de ce système, il est possible de mesurer la réflectivité de miroir multicouches, les réponses en longueur d'onde de photocathodes de caméras ultra rapides (streak cameras) et d'autres détecteurs de photons tels que caméras $\mathrm{CCD}$ en illumination directe, films photographiques, etc....

Ces mesures sont faites à l'aide de photodiodes à cathode en aluminium pur dont on connait la réponse en absolu dans la gamme de longueurs d'onde correspondant à la zone de travail.

\section{Description du dispositif.}

Le schéma du système d'étalonnage est représenté sur la figure 1.

\section{$\underline{\text { I.1 Le laser }}$}

Un laser de puissance pulsé est focalisé sur une cible solide métallique. Ce laser délivre une énergie de 10 joules en $4 \mathrm{~ns}$ à la longueur d'onde de $1.054 \mu \mathrm{m}$. Il est doublé en fréquence avec un rendemment de $50 \%$. L'action du laser sur la cible crée un plasma qui emet un rayonnement sur une large bande allant du visible aux rayons $\mathrm{X}$. En effet, à cause de sa densité et de sa température élevées $\left(>10^{21} \mathrm{~cm}^{-3}\right.$ et $\left.1 \mathrm{keV}\right)$, un tel plasma est le siège d'un taux de conversion $X$ élevé.

Nous présentons à titre d'exemple le spectre d'émission de plasmas créés sur aluminium (figure 2) et or (figure 3) par un laser de puissance donnant un éclairement au foyer de $2.10^{14} w / \mathrm{cm}^{2}$ à $0.26 \mu \mathrm{m}$ de longueur d'onde[1]. On constate que dans la partie qui nous intéresse (quelques eV à quelques centaines d'eV) le spectre est très intense quelque soit le numéro atomique du matériau 


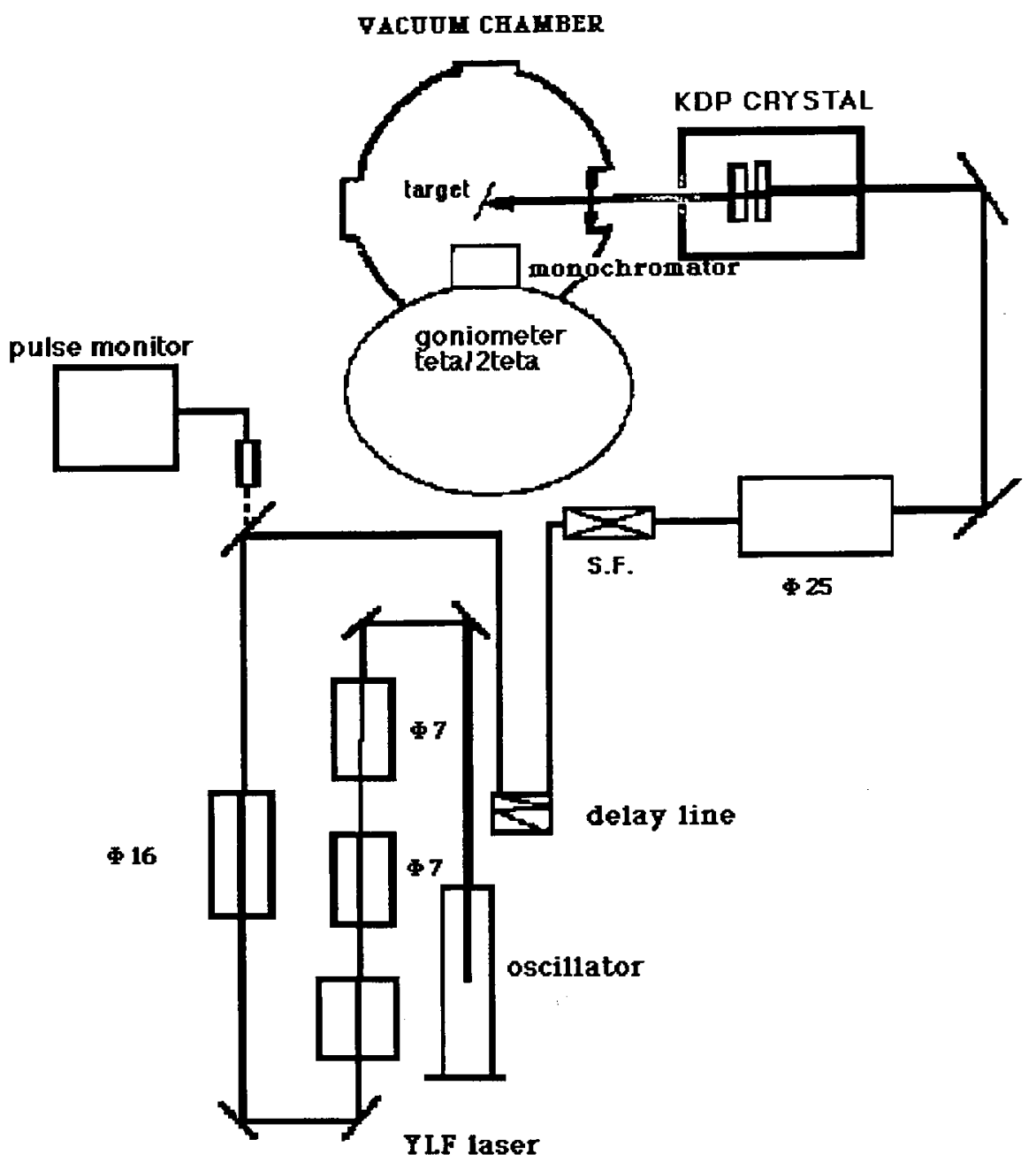

Figure 1. Schéma général du système d'étalonnage

\section{I.2 le monochromateur}

Le plasma servant de source de rayonnement est observé par un monochromateur [2] dont le sché ma est représenté sur la figure 4. Pour balayer la gamme de longueurs d'onde $40 \AA$ $-400 \AA$, trois réseaux sont utilisés 


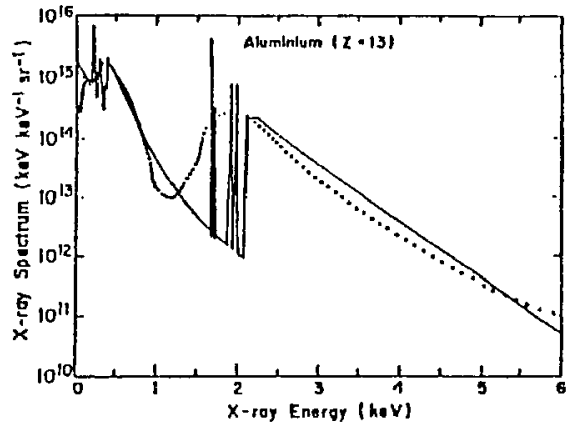

Figure 2

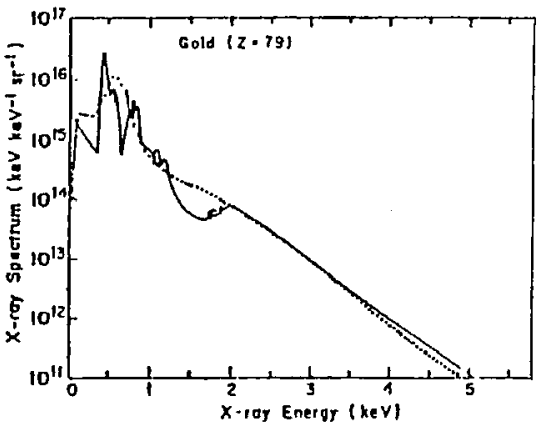

Figure3

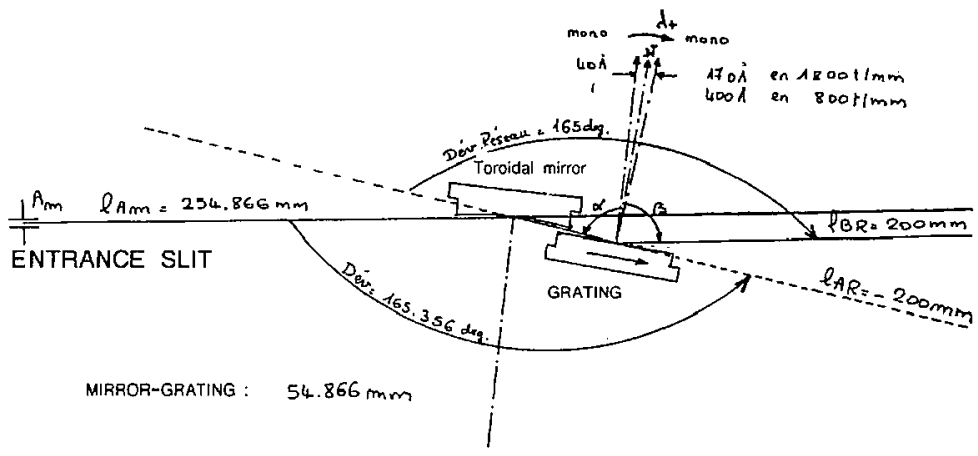

Figure 4

Le premier réseau a 1800 traits par millimètre. Il permet de travailler entre $35 \AA$ et $200 \AA$. Le deuxième a 800 traits par millimètres et le demier 400 traits par millimètres, ce qui permet d'étendre le spectre vers les grandes longueurs d'onde jusqu'à $800 \AA$. Un miroir torique est mis en avant des réseaux. Il donne une image de la fente d'entrée de grandissement 1 dans le plan de la fente de sortie sélectionnant les longueurs d'onde.

Les courbes d'efficacité du monochromateur pour chacun des réseaux sont représentées sur la figure 5. La courbe correspondant à Rplatine donne la réflectivité du mirroir torique dont il a été tenu compte pour déterminer les efficacités.

\section{I.3 Le réflectomètre}

La lumière sélectionné par le monochromateur est envoyée dans une enceinte à vide contenant un goniomètre $\theta / 2 \theta$ servant à faire les différents étalonnages.

Le schéma du goniomètre est représenté sur la figure 6. 


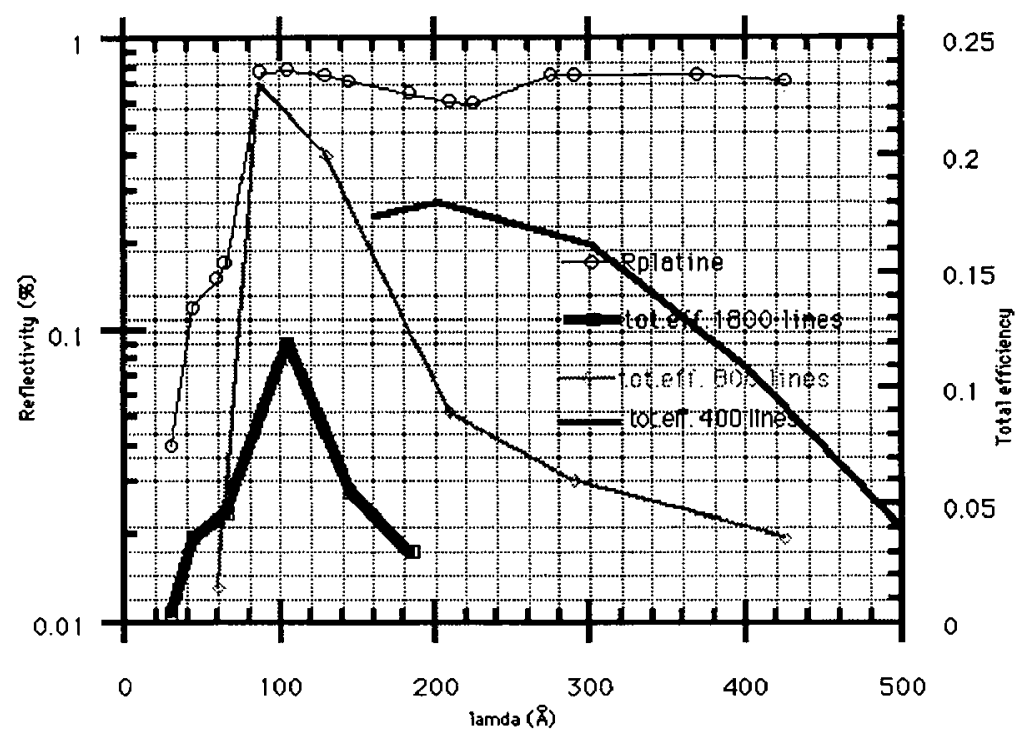

Figure 5. Efficacité du monochromateur

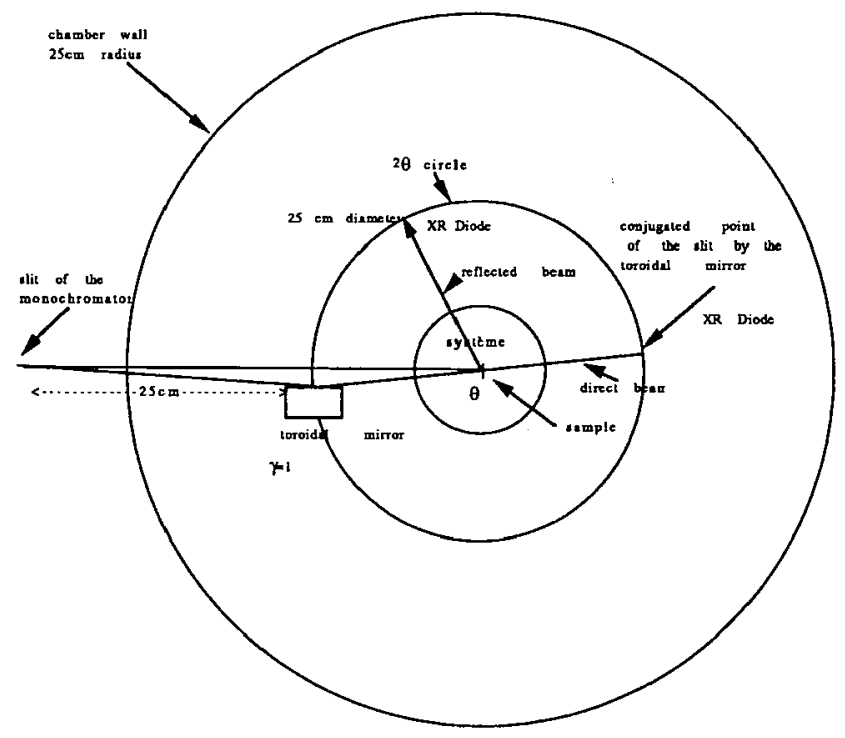

Figure 6. Le goniomètre $\theta / 2 \theta$ 
Un miroir torique identique au miroir du monochromateur forme limage de la fente de sortie du monochromateur sur le cercle $2 \theta$ du goniomètre. Sur ce cercle, sont placées deux photodiodes (XRD) servant à détecter les signaux: une diode fixe dans l'axe du système permet d'enregistrer le signal direct, une diode mobile enregistre le signal dévié par un échantillon placé au centre du goniomètre. Cette dernière diode est asservie au mouvement de l'échantillon placé au centre et tourne de $2 \theta$ lorsque l'échantillon est roté d'un angle $\theta$.

\section{I.4 Les photodiodes}

Ces photodiodes que nous appellerons XRD pour $\mathrm{x}$-ray diodes ont une cathode en aluminium pur dont la sensibilité en fonction de la longueur d'onde est donnée dans la reférence 3.

La tension aux bornes d'une résistance $\mathrm{R}(50 \Omega$ dans notre cas) générée par des photons arrivant sur la photocathode de la diode est:

$$
\mathbf{V}=\mathbf{R} \int_{0}^{-} F(\lambda) S(\lambda) L(\lambda) A \Omega \mathbf{d} \lambda
$$

F est la transmission d'un filtre s'il y en a placé en entrée de diode

$S$ est la sensibilité spectrale de la cathode en coulomb/joule

L est la densité spectrale en watts/stéradians $/ \mathrm{m}^{2}$ proportionnelle au nombre de photons à la longueur d'onde $\lambda$

A est la surface de la source en $\mathrm{m}^{2}$

$\Omega$ est l'angle solide de la photocathode.

Dans notre cas nous avons un rayonnement monochromatique. Il n'y a donc pas d'intégration

Pour la mesure de réflectivité de miroir X-UV à longueur d'onde donnée en fonction de l'incidence, on compare le signal réfléchi au signal direct. Dans ce cas le rapport des signaux est égal directement à la réflectivité.

Pour la mesure de la réponse en longueur d'onde il faut en plus tenir compte de S. Les autres paramètre n'ont pas à être pris en compte.

\section{Procédures de "calibration" assistées par ordinateur}

Un étalonnage nécéssitant une variation d'angle d'incidence et une variation de longueur d'onde du rayonnement doit être automatique. Nous avons choisi d'utiliser le logiciel Labview de National Instruments tournant sur un MacII Fx d'Apple pour gérer l'expérience.

Deux cartes d'interface de National Instruments reconnaissant Labview sont utilisées pour cela: Une carte GPIB qui sert à commander le moteur du réseaux du monochromateur et qui permet ainsi de selectionner la longueur d'onde de travail. Une carte NB-MIO-16 permet de numeriser et d'acquérir les signaux de diodes. Les moteurs d'entrainement des mouvements pas à pas du goniomètre sont gerés par un automate de Microcontrole luimême commandé par l'ordinateur à travers sa sortie serie.

Ainsi on peut effectuer les mesures suivantes:

*- Réflectivité de miroirs ou de réseaux : L'échantillon est placé en autocollimation avec le faisceau incident qui donne la référence (incidence 0 ). Il est ensuite tourné vers la valeur de l'incidence pour laquelle il a été construit diminuée de $20^{\circ}$. 
-Longueur d'onde fixée \& $R=$ f(angle)

$-1^{\circ}$ ) On entre la valeur de la longueur d'onde. Le logiciel Labview fait tourner le réseau du monochromateur jusqu'à ce que la longueur d'onde désirée passe par la fente du monochromateur.

$-2^{\circ}$ ) Le laser crée le plasma et envoie un signal de déclenchement qui met en route la numérisation et sauvegarde le signal des diodes XRD.

$\left.-3^{\circ}\right)$ La cible est déplacée automatiquement après chaque tir laser et le tir est répété pour le même angle d'incidence. Le signal numérisé est comparé avec le précédent. Si sa valeur diffère de moins de $10 \%$ par rapport au signal précédent, il est numérisé et la valeur moyenne des deux signaux est prise en compte; le miroir est tourné de $1^{\circ}$ d'angle et le détecteur (XRD) de $2^{\circ}$. Si ce n'est pas le cas, la dernière valeur numérisée n'est pas prise en compte et le tir laser est répété sans tourner l'échantillon et la diode jusqu'à ce que le bon signal soit obtenu.

$-4^{\circ}$ ) Lorsqu'une valeur moyenne acceptable du signal réfléchi est mise en mémoire, l'échantillon est dégagé automatiquement et le flux direct de photons X-UV est mesuré par la diode directe afin d'enregistrer la référence. La procédure est répétée comme pour le signal réfléchi.

$\left.-5^{\circ}\right)$ L'échantillon est remis en place et le cycle de mesures est répété dans l'intervalle d'angle d'incidences déterminé préalablement.

\section{-Angle fixé \& $R$ = f(longueur d'onde)}

Même procédure que précédemment mais l'échantillon n'est pas tourné, c'est le réseau du monochromateur qui l'est pour faire varier la longueur d'onde.

*-Autres possibilités : mesure des réponses en longueur d'onde de photocathodes de streaks et autres détecteurs photosensibles tels que caméras $\mathrm{CCD}$ etc...

\section{Application à la réponse d'un CCD aminci en illumination directe.}

En conclusion et comme première application de l'utilisation du système, nous avons mesuré la réponse relative d'un $\mathrm{CCD}$ aminci attaqué par l'arrière en fonction de la longueur d'onde. Cette mesure a été effectuée pour le comptedu CEA Limeil qui possède un tel composant, monté sur une caméra permettant des acquisitions directes d'images. Le chip CCD est du type Thomson TXHX31164.

Nous avons mesuré le niveau de gris moyen de l'image de la fente d'entrée faite sur le CCD en fonction de la longueur d'onde entre $100 \AA$ et $200 \AA$. Compte tenu de la réponse de la cathode, nous avons mesuré l'intensité relative incidente à l'aide d'une photodiode. Ainsi nous avons tracé sur la figure 7 les points de mesure expérimentaux et la courbe théorique correspondant à un susbtrat de silcium de $100 \AA$ d'épaisseur et un champ électrique dans le composant de $10^{3} \mathrm{~V} / \mathrm{cm}$. Il apparait une bonne concordance entre les point expérimentaux et la courbe calculée. Le flanc d'absorption $\mathrm{L} 1$ du silicium à $105,05 \mathrm{eV}$ est bien mis en évidence par la mesure. 


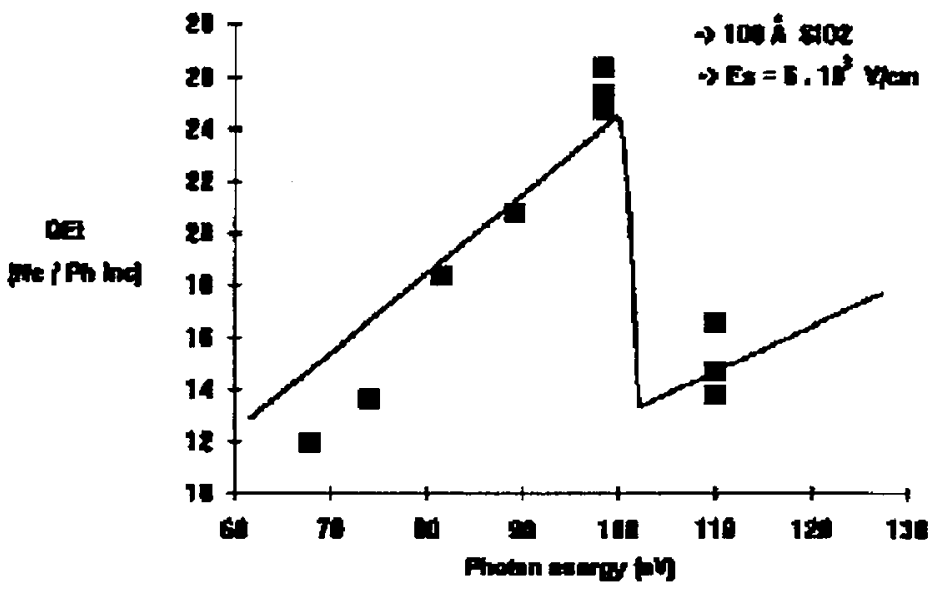

Figure 7. Courbe d'étalonnage du CCD aminci Thomson THX31164

\section{Références}

1.R. Fabbro et al. Phys. Rev. A 34,4184,(1987)

2. R. Benattar et A. Sezen, X-Ray lasers 90 , Proceedings of the 2nd International Colloquium, Institute of Physics conferences series number 116, p65 (1991)

3. Day \& Saloman, J. Appl. Phys., 52,6965, (1981). 